\title{
Dynamic response of a beam resting on a nonlinear foundation to a moving load: Coiflet-based solution
}

\author{
Piotr Koziol* and Zdzislaw Hryniewicz \\ Department of Civil and Environmental Engineering, Koszalin University of Technology, Koszalin, Poland
}

\begin{abstract}
This paper presents a new semi-analytical solution for the Timoshenko beam subjected to a moving load in case of a nonlinear medium underneath. The finite series of distributed moving loads harmonically varying in time is considered as a representation of a moving train. The solution for vibrations is obtained by using the Adomian's decomposition combined with the Fourier transform and a wavelet-based procedure for its computation. The adapted approximating method uses wavelet filters of Coiflet type that appeared a very effective tool for vibration analysis in a few earlier papers. The developed approach provides solutions for both transverse displacement and angular rotation of the beam, which allows parametric analysis of the investigated dynamic system to be conducted in an efficient manner. The aim of this article is to present an effective method of approximation for the analysis of complex dynamic nonlinear models related to the moving load problems.
\end{abstract}

Keywords: Moving load, Timoshenko beam, nonlinear vibrations, wavelet approximation, Adomian's decomposition

\section{Introduction}

A rapidly growing development of train transportation and its influence on human environment lead to new problems concerning prevention of surroundings against negative factors coming from dynamic excitations. New modelling approaches and methods of solution for vibration analysis are needed and this fact is highlighted by both the industry and scientists. In reality, foundation for rail constructions is highly nonlinear and therefore models taking into account nonlinear properties of real structures become an important subject of modern investigations.

Many interesting results concerning moving load problems, in mostly linear cases, can be found in the literature [1]. The nonlinear models are still relatively undiscovered and open for investigation. Some results were obtained by using numerical simulations, showing better modelling possibilities when nonlinear systems are considered. Dahlberg [2] showed that the nonlinear model simulates the beam deflection better than the linear models in relation with measurements. A similar nonlinear model was used by $\mathrm{Wu}$ and Thompson [3] for the analysis of dynamic response using FEM. Only a small number of papers presenting analytical approaches were published, highlighting their importance for the correct analysis of nonlinearity. Especially interesting is the paper [4], where the governing nonlinear equations of motion for Timoshenko beam are solved by using a perturbation method in conjunction with the Fourier transform and the Cauchy's residue theorem. Nevertheless, the obtained solution gives only partial characteristics of the system, it is inefficient for parametric analysis and highly doubtful in some areas due to the complexity of the applied residues calculation method along with growing complications related to a higher order of approximation based on the perturbation approach.

*Corresponding author: Piotr Koziol, Department of Civil and Environmental Engineering, Koszalin University of Technology, Sniadeckich 2, 75-453 Koszalin, Poland. E-mail: piotr.koziol@wbiis.tu.koszalin.pl. 
The present paper describes a new semi-analytical approach for the analysis of the dynamic response of Timoshenko beam resting on a nonlinear elastic foundation. For a better representation of a moving train, the finite series of distributed moving loads harmonically varying in time is considered [5]. The nonlinear differential Timoshenko beam equations with a hysteretic or viscous damping of foundation are considered [4] for the representation of a railway track structure. It is known that the Timoshenko beam gives more reliable results when one deals with long tracks, especially in case of high velocities and frequencies [6].

The Galilean co-ordinate system is applied in order to obtain the steady-state response of the beam. The Adomian's decomposition method [7] combined with a wavelet-based approximation of functions [8] gives solution for the transverse displacement and rotation. The adopted approximation using Coiflet filters allows an effective parametric analysis of the system in the area of solutions' stability. The wavelet approximation is used instead of a direct numerical integration of the Fourier integrals, leading to solutions without errors appearing in previous results. Various systems of parameters describing physical properties of the structure are used for the numerical examples in order to show a significant influence of a nonlinear factor on a dynamic behaviour of the system.

The novelty of the article is represented by the new approach for analysis of dynamic nonlinear system with the considered type of load and a new semi-analytical solution for vibrations of infinite Timoshenko beam resting on a nonlinear viscoelastic medium. The investigated model of a beam with the applied load reflects well enough, in some areas, interactions appearing in real structures. It is, together with a solution, an important contribution to the subject of railway structures analysis.

\section{Equations and conditions}

The Timoshenko beam has a specific form taking into account both the shear deformations and the rotary inertia of cross sections.

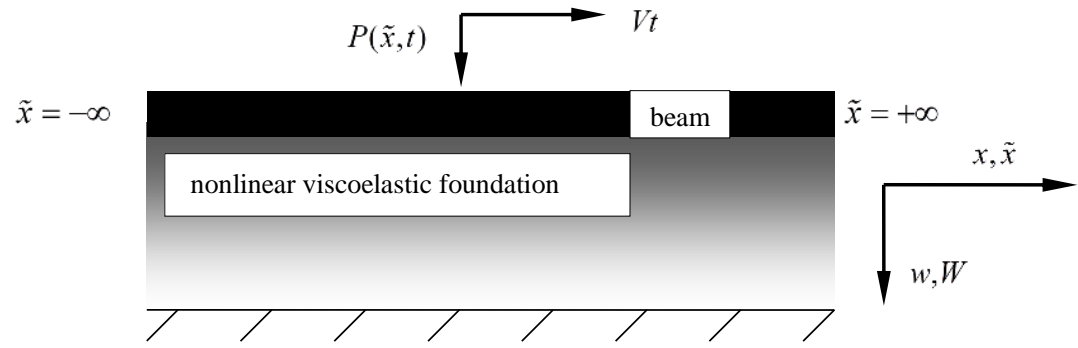

Fig. 1. The beam resting on a nonlinear viscoelastic foundation.

The dynamic equations for a homogeneous infinite beam on a nonlinear viscoelastic foundation (Fig. 1) can be written as follows:

$$
\begin{aligned}
& m_{b} \frac{\partial^{2} W}{\partial t^{2}}+C_{d} \frac{\partial W}{\partial t}-S \frac{\partial^{2} W}{\partial \tilde{x}^{2}}+S \frac{\partial \Psi}{\partial \tilde{x}}+k_{L} W+k_{N} W^{3}=-P(\tilde{x}, t) \\
& J \frac{\partial^{2} \Psi}{\partial t^{2}}-E I \frac{\partial^{2} \Psi}{\partial \tilde{x}^{2}}+S \Psi-S \frac{\partial W}{\partial \tilde{x}}=0
\end{aligned}
$$

where $W$ is the transverse displacement, $\Psi$ is the angular rotation of the cross-section, $\tilde{x}$ is the space coordinate in a direction along the beam, $t$ represents the time, $k_{N}$ is a nonlinear part of foundation stiffness, $k_{L}$ is the linear coefficient of foundation stiffness, $k_{L}=\tilde{k}(1+2 \zeta i), i=\sqrt{-1}, \zeta$ is the hysteretic damping ratio and the parameters are: the Young's modulus $E$, the shear modulus $G$, the mass density $\rho$, the viscous damping of foundation $C_{d}$, the cross-section area $A$, the moment of inertia $I$, the shear correction factor $\kappa$, the mass per unit length $m_{b}=\rho A$, the shear stiffness $S=\kappa A G$, the beam flexural rigidity $E I$, the mass moment of inertia $J=\rho I$. The solution for 
the infinite beam must be determined in such way that the displacement, the bending moment, the shear force and the slope of the beam curvature tend to zero, and these properties should be reflected in boundary conditions.

In this paper, a finite sum of distributed harmonically varying in time loads is considered (Fig. 2), giving the possibility of representation of an excitation generated by a train consisting of a few wagons:

$$
P(\tilde{x}, t)=\sum_{l=0}^{L-1} \frac{P_{0}}{2 r} \cos ^{2}\left(\frac{\pi(\tilde{x}-V t-(2 r+s) l)}{2 r}\right) H\left(r^{2}-(\tilde{x}-V t-(2 r+s) l)^{2}\right) e^{i \Omega t} .
$$

$H(),. 2 r, V$ and $\Omega=2 \pi f_{\Omega}$ are the Heaviside function, the span of load, the velocity and the frequency of the moving load, respectively. $\frac{P_{0}}{2 r}$ is the value of the amplitude for the load, $L$ is a number of separated impulses and $s$ is the distance between them.

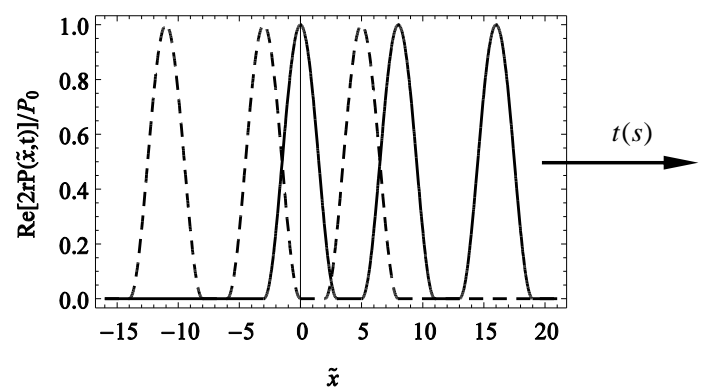

Fig. 2. The series of distributed harmonic loads for $s=2 \mathrm{~m}, r=3 \mathrm{~m}, V=10 \mathrm{~m} / \mathrm{s}, L=3, f_{\Omega}=0 \mathrm{~Hz}$ : $t=-1.1 \mathrm{~s}$ (dashed line), $t=0 \mathrm{~s}$ (solid line).

In order to analyse the steady-state response, one can use the Galilean co-ordinate transformation $x=\tilde{x}-V t$. The following representation of solution:

$$
W(x, t)=w(x) e^{i \Omega t}=w(\tilde{x}-V t) e^{i \Omega t}, \Psi(x, t)=\psi(x) e^{i \Omega t}=\psi(\tilde{x}-V t) e^{i \Omega t}
$$

is used in further computations for a sake of simplicity. The use of the chain rule applied to those formulas leads to following expressions:

$$
\begin{aligned}
& \frac{\partial W}{\partial \tilde{x}}=\frac{d w}{d x} e^{i \Omega t}, \frac{\partial^{2} W}{\partial \tilde{x}^{2}}=\frac{d^{2} w}{d x^{2}} e^{i \Omega t}, \frac{\partial W}{\partial t}=\left(-V \frac{d w}{d x}+i \Omega w\right) e^{i \Omega t}, \frac{\partial^{2} W}{\partial t^{2}}=\left(V^{2} \frac{d^{2} w}{d x^{2}}-2 i \Omega V \frac{d w}{d x}-\Omega^{2} w\right) e^{i \Omega t} \\
& \frac{\partial \Psi}{\partial \tilde{x}}=\frac{d \psi}{d x} e^{i \Omega t}, \frac{\partial^{2} \Psi}{\partial \tilde{x}^{2}}=\frac{d^{2} \psi}{d x^{2}} e^{i \Omega t}, \frac{\partial \Psi}{\partial t}=\left(-V \frac{d \psi}{d x}+i \Omega \psi\right) e^{i \Omega t}, \frac{\partial^{2} \Psi}{\partial t^{2}}=\left(V^{2} \frac{d^{2} \psi}{d x^{2}}-2 i \Omega V \frac{d \psi}{d x}-\Omega^{2} \psi\right) e^{i \Omega t}
\end{aligned}
$$

and, consequently, to the system of equations:

$$
\begin{aligned}
& a_{0} \frac{d^{2} w}{d x^{2}}+a_{1} \frac{d w}{d x}+a_{2} w+S \frac{d \psi}{d x}=-P(x) e^{-i \Omega t}-k_{N} w^{3} e^{2 i \Omega t}, \\
& b_{0} \frac{d^{2} \psi}{d x^{2}}+b_{1} \frac{d \psi}{d x}+b_{2} \psi-S \frac{d w}{d x}=0
\end{aligned}
$$

with the new coefficients: $a_{0}=m_{b} V^{2}-S, \quad a_{1}=-V\left(2 i \Omega m_{b}+C_{d}\right), \quad a_{2}=k_{L}-m_{b} \Omega^{2}+i C_{d} \Omega, \quad b_{0}=J V^{2}-E I$, $b_{1}=-2 i \Omega V J$ and $b_{2}=S-\Omega^{2} J$. Then by defining the differential operators: 


$$
L_{a}=a_{0} \frac{d^{2}}{d x^{2}}+a_{1} \frac{d}{d x}+a_{2} \text { and } L_{b}=b_{0} \frac{d^{2}}{d x^{2}}+b_{1} \frac{d}{d x}+b_{2}
$$

one can reformulate Eqs (6) and (7):

$$
L_{a} w+S \frac{d \psi}{d x}=-P(x) e^{-i \Omega t}-k_{N} w^{3}(x) e^{2 i \Omega t}, L_{b} \psi-S \frac{d w}{d x}=0 .
$$

\section{Adomian's decomposition}

The Adomian's decomposition is a very efficient method of solution for both stochastic and nonlinear models, which has been used successfully in the analysis of many dynamic systems [7-10]. This approach is used for a nonlinear differential equation and assumes a specific form of solution represented by a series with one linear term and infinite number of functions related to the nonlinear part that must be found in order to solve the system. In case of Eq. (6), the nonlinear part can be represented by a series

$$
w^{3}(x)=\sum_{j=0}^{\infty} Q_{j}(x)
$$

where $Q_{j}(x)$ are the Adomian polynomials. They can take the following form [9]:

$$
Q_{j}(x)=\frac{1}{j !}\left[\frac{d^{j}}{d \lambda^{j}}\left(\sum_{k=0}^{\infty} \lambda^{k} w_{k}(x)\right)^{3}\right]_{\lambda=0} \text { for } j=0,1,2, \ldots .
$$

According to the Adomian's approximation theory, one can assume the specific form of solution:

$$
w(x)=\sum_{j=0}^{\infty} w_{j}(x) \text { and } \psi(x)=\sum_{j=0}^{\infty} \psi_{j}(x) .
$$

Hence the explicit forms of the Adomian polynomials can be determined as follows [9]:

$$
\begin{aligned}
& Q_{0}=w_{0}^{3}, Q_{1}=3 w_{0}{ }^{2} w_{1}, Q_{2}=3\left(w_{0} w_{1}{ }^{2}+w_{0}{ }^{2} w_{2}\right), Q_{3}=w_{1}^{3}+6 w_{0} w_{1} w_{2}+3 w_{0}{ }^{2} w_{3}, \\
& Q_{4}=3\left(w_{1}^{2} w_{2}+w_{0} w_{2}^{2}+2 w_{0} w_{1} w_{3}+w_{0}{ }^{2} w_{4}\right), \ldots
\end{aligned}
$$

\section{Solution in transform domain}

In order to secure the convergence of the solution represented by an Adomian series, the following conditions must be fulfilled [11]:

$$
0 \leqslant \alpha_{j}^{w}<1 \text { and } 0 \leqslant \alpha_{j}^{\psi}<1 \text { for } j=0,1,2, \ldots
$$

where the parameters $\alpha_{j}^{w}$ and $\alpha_{j}^{\psi}$ are defined as follows:

$$
\alpha_{j}^{w}=\left\{\begin{array}{ll}
\left\|w_{j+1}\right\| /\left\|w_{j}\right\|, & \left\|w_{j}\right\| \neq 0 \\
0, & \left\|w_{j}\right\|=0
\end{array}, \quad \alpha_{j}^{\psi}= \begin{cases}\left\|\psi_{j+1}\right\| /\left\|\psi_{j}\right\|, & \left\|\psi_{j}\right\| \neq 0 \\
0, & \left\|\psi_{j}\right\|=0\end{cases}\right.
$$


with the norm $\|f(x)\|=\max _{x}|f(x)|=\max _{x} \sqrt{\operatorname{Re}[f(x)]^{2}+\operatorname{Im}[f(x)]^{2}}$. The effective analysis of the solution can be carried out, under conditions defined by Eq. (14), by using the $n$-th order approximation $S_{n}{ }^{w}(x)$ and $S_{n}{ }^{\psi}(x)$ with a relatively small $n$, where

$$
S_{n}^{w}(x)=\sum_{j=0}^{n} w_{j}(x) \text { and } S_{n}^{\psi}(x)=\sum_{j=0}^{n} \psi_{j}(x) .
$$

The classical Fourier transform can be used for analysis of the steady-state solution:

$$
\begin{aligned}
& \hat{w}(\omega)=F[w(x)]=\int_{-\infty}^{\infty} w(x) e^{-i \omega x} d x, w(x)=F^{-1}[\hat{w}(\omega)]=\frac{1}{2 \pi} \int_{-\infty}^{\infty} \hat{w}(\omega) e^{i \omega x} d \omega, \\
& \hat{\psi}(\omega)=F[\psi(x)]=\int_{-\infty}^{\infty} \psi(x) e^{-i \omega x} d x, \psi(x)=F^{-1}[\hat{\psi}(\omega)]=\frac{1}{2 \pi} \int_{-\infty}^{\infty} \hat{\psi}(\omega) e^{i \omega x} d \omega .
\end{aligned}
$$

Formulas (9-11) lead to the following system of equations:

$$
\begin{aligned}
& L_{a} w_{0}+S \frac{d \psi_{0}}{d x}=-P(x) e^{-i \Omega t}, L_{b} \psi_{0}-S \frac{d w_{0}}{d x}=0, \\
& L_{a} w_{j}+S \frac{d \psi_{j}}{d x}=-k_{N} Q_{j-1}(x) e^{2 i \Omega t}, L_{b} \psi_{j}-S \frac{d w_{j}}{d x}=0
\end{aligned}
$$

for $j=1,2, \ldots$ The terms $w_{j}$ and $\psi_{j}$ are determined by applying recursively Eqs (18a, b).

The transformed Eqs (18a, b) give solutions for $w_{j}$ and $\psi_{j}$ in the transform domain:

$$
\begin{aligned}
& \hat{w}_{0}(\omega)=\hat{P}(\omega) H_{b}(\omega) /\left(S^{2} \omega^{2}-H_{a}(\omega) H_{b}(\omega)\right), \hat{\psi}_{0}=i S \omega \hat{P}(\omega) /\left(S^{2} \omega^{2}-H_{a}(\omega) H_{b}(\omega)\right), \\
& \hat{w}_{j}(\omega)=\frac{k_{N} H_{b}(\omega) \hat{Q}_{j-1}(\omega)}{S^{2} \omega^{2}-H_{a}(\omega) H_{b}(\omega)} e^{2 i \Omega t}, \hat{\psi}_{j}(\omega)=\frac{i S k_{N} \omega \hat{Q}_{j-1}(\omega)}{S^{2} \omega^{2}-H_{a}(\omega) H_{b}(\omega)} e^{2 i \Omega t},
\end{aligned}
$$

for $j=1,2, \ldots$, where

$$
H_{a}(\omega)=-a_{0} \omega^{2}+i a_{1} \omega+a_{2}, H_{b}(\omega)=-b_{0} \omega^{2}+i b_{1} \omega+b_{2}
$$

and

$$
\hat{P}(\omega)=P_{0} \frac{i \pi^{2}(\exp (2 i r \omega)-1)}{4 r \omega\left(\pi^{2}-r^{2} \omega^{2}\right)} \sum_{l=0}^{L-1} \exp (-i(r+2 l r+l s) \omega) .
$$

The inverse Fourier transform of Eqs (19a, b) leads to the solution in the physical domain. The derivation of the Fourier transform of the Adomian polynomials $Q_{j}(x)$ and the inverse Fourier transform of the terms $\hat{w}_{j}(\omega)$ and $\hat{\psi}_{j}(\omega)$ is difficult due to a specific form of integrands. Classical methods of calculation and the direct numerical integration are ineffective and do not give solutions precise enough for the parametric analysis, especially when one must use the higher order of Adomian's approximation. One of alternative methods of Fourier integrals calculation is an approximation of functions based on a wavelet theory. This method was previously used for analysis of many dynamic systems related to moving load problems, leading to new solutions that can be used within the context of parametric-type studies $[8,10,12]$. 


\section{Wavelet approximation}

The wavelet method adopted in this paper is based on a wavelet expansion of functions by using multiresolution coefficients (wavelet coefficients) [13,14]:

$$
\begin{aligned}
& c_{n, k}=\left\langle f, \Phi_{n, k}\right\rangle=\int_{-\infty}^{+\infty} f(x) \Phi_{n, k}(x) d x, \\
& d_{j, k}=\left\langle f, \Psi_{j, k}\right\rangle=\int_{-\infty}^{+\infty} f(x) \Psi_{j, k}(x) d x
\end{aligned}
$$

that are an inner product of a given function $f(x)$ and elements of wavelet bases

$$
\begin{aligned}
& \left\{\Phi_{n, k}(x)=2^{n / 2} \Phi\left(2^{n} x-k\right)\right\}_{n, k}, \\
& \left\{\Psi_{j, k}(x)=2^{j / 2} \Psi\left(2^{j} x-k\right)\right\}_{j, k}
\end{aligned}
$$

generated by dilation and translation from scaling function $\Phi$ and wavelet function $\Psi$, respectively. In order to apply the approximation algorithms, one must evaluate integrals $(21 \mathrm{a}, \mathrm{b})$. They are usually difficult to compute but can be relatively easy approximated in case of Coiflets $[15,16]$, the family of wavelet filters constructed specially for numerical applications. The wavelet and scaling functions defined by Coiflets:

$$
\Psi^{C}(x)=\sum_{k=0}^{3 N-1}(-1)^{k} p_{3 N-1-k} \Phi^{C}(2 x-k), \Phi^{C}(x)=\sum_{k=0}^{3 N-1} p_{k} \Phi^{C}(2 x-k)
$$

where $N$ is treated as a degree of accuracy of the Coiflet filter $\left(p_{k}\right)$, possess the property of vanishing moments $[13,16]$ allowing to estimate analytically the wavelet coefficients for functions $f(x) \in L^{2}(\boldsymbol{R})$. It can be shown [14] that:

$$
\begin{aligned}
& c_{n, k}=\int_{-\infty}^{+\infty} f(x) \Phi_{n, k}^{C}(x) d x \approx 2^{-n / 2} f\left((k+M) 2^{-n}\right), \\
& d_{j, k}=\int_{-\infty}^{+\infty} f(x) \Psi_{j, k}^{C}(x) d x \approx 2^{-j / 2-1} \sum_{m=0}^{3 N-1}(-1)^{m} p_{3 N-1-m} f\left((M+m+2 k) 2^{-j-1}\right)
\end{aligned}
$$

where $M=\sum_{k=0}^{3 N-1} k p_{k}, \Phi_{n, k}^{C}(x)=2^{n / 2} \Phi^{C}\left(2^{n} x-k\right)$ and $\Psi_{j, k}^{C}(x)=2^{j / 2} \Psi^{C}\left(2^{j} x-k\right)$. Formulas (24a, b) lead to the effective approximation of the Fourier transform and the inverse Fourier transform:

$$
\begin{gathered}
\hat{f}(\omega)=2^{-n / 2} \hat{\Phi}^{C}\left(\omega / 2^{n}\right) \sum_{k=-\infty}^{+\infty} c_{n, k} \exp \left(-i \omega k / 2^{n}\right)+\sum_{j=n}^{\infty} 2^{-j / 2} \hat{\Psi}^{C}\left(\omega / 2^{j}\right) \sum_{k=-\infty}^{+\infty} d_{j, k} \exp \left(-i \omega k / 2^{j}\right), \\
f(x)=\left(2^{-n / 2} / 2 \pi\right) \hat{\Phi}^{C}\left(-x / 2^{n}\right) \sum_{k=-\infty}^{+\infty} c_{n, k} \exp \left(i x k / 2^{n}\right)+ \\
+\frac{1}{2 \pi} \sum_{j=n}^{\infty} 2^{-j / 2} \hat{\Psi}^{C}\left(-x / 2^{j}\right) \sum_{k=-\infty}^{+\infty} d_{j, k} \exp \left(i x k / 2^{j}\right) .
\end{gathered}
$$


The Coiflet filter $\left(p_{k}\right)$ used in this paper [16] gives an efficient algorithm for calculation of transforms (Eqs (17a, b)) $[8,14]$

$$
\begin{aligned}
& \hat{f}(\omega)=\lim _{n \rightarrow \infty} \hat{f}_{n}(\omega)=\lim _{n \rightarrow \infty} 2^{-n} \prod_{k=1}^{\infty}\left(\sum_{j=0}^{3 N-1} p_{k} e^{-i j \omega / 2^{n+k}}\right) \sum_{k=-\infty}^{+\infty} f\left((k+M) 2^{-n}\right) e^{-i \omega k 2^{-n}} \\
& f(x)=\lim _{n \rightarrow \infty} f_{n}(x)=\lim _{n \rightarrow \infty} \frac{1}{2^{n+1} \pi} \prod_{k=1}^{\infty}\left(\sum_{j=0}^{3 N-1} p_{j} e^{i j x / 2^{n+k}}\right) \sum_{k=-\infty}^{+\infty} \hat{f}\left((k+M) 2^{-n}\right) e^{i x k 2^{-n}} .
\end{aligned}
$$

A good balance between efficiency and cost-effectiveness must be found when applying the Coiflet-based approximation. The approximation order should be chosen on the basis of stabilisation of solutions with increasing parameter $n$ [8]. Numerical simulations show that it is enough to assume $n=5$ for the analysed system, i.e. one can take $\hat{f}(w) \approx \hat{f}_{5}(w)$ and $f(x) \approx f_{5}(x)$ in Eqs $(26)$ and (27).

\section{Coiflet-based solution}

Formula (27) applied to (Eqs (19a, b)) gives the following representation of the approximated solution:

$$
\begin{aligned}
& w_{j}(x)=\frac{1}{2^{n+1} \pi} \prod_{k=1}^{k_{p}} \sum_{j=0}^{3 N-1} p_{j} \exp \left(j i x 2^{-n-k}\right) \sum_{k=k_{\min }}^{k_{\max }} \hat{w}_{j}\left((k+M) 2^{-n}\right) \exp \left(i k x 2^{-n}\right), \\
& \psi_{j}(x)=\frac{1}{2^{n+1} \pi} \prod_{k=1}^{k_{p}} \sum_{j=0}^{3 N-1} p_{j} \exp \left(j i x 2^{-n-k}\right) \sum_{k=k_{\min }}^{k_{\max }} \hat{\psi}_{j}\left((k+M) 2^{-n}\right) \exp \left(i k x 2^{-n}\right) .
\end{aligned}
$$

where $k_{p}$ is a parameter associated with the used Coiflet filter and an order of approximation $n$. The Fourier transforms of Adomian polynomials appearing in $\hat{w}_{j}(\omega)$ and $\hat{\psi}_{j}(\omega)$ (Eq. (19b)) are derived by using estimation (Eq. (26)):

$$
\hat{Q}_{j}(\omega)=2^{-n} \prod_{k=1}^{k_{p}} \sum_{j=0}^{3 N-1} p_{j} \exp \left(-j i \omega 2^{-n-k}\right) \sum_{k=k_{\min }}^{k_{\max }} Q_{j}\left((k+M) 2^{-n}\right) \exp \left(-i k \omega 2^{-n}\right) .
$$

The range of summation $k_{\min }=\omega_{\min } 2^{n}-3 N+2$ and $k_{\max }=\omega_{\max } 2^{n}-1$ can be determined on the basis of the information about the investigated function, i.e. the interval $\left[\omega_{\min }, \omega_{\max }\right]$ must cover the set of points having a strong influence on the sought function behaviour [8,14]. It should contain all points for which the values of the transformed solution are significantly different from zero [8].

\section{Numerical examples}

The adopted wavelet-based approximation method was previously validated by simpler standard problems [8]. It was also used for more complex models related to moving loads analysis [5,10,12,17].

The following system of parameters $[2,4,17-19]$ is used for numerical simulations carried out in this paper: $\zeta=0.02, \quad k_{N}=5 \cdot 10^{11} \mathrm{~N} / \mathrm{m}^{4}, \tilde{k}=2 \cdot 10^{7} \mathrm{~N} / \mathrm{m}^{2}, P_{0}=8 \cdot 10^{4} \mathrm{~N}, E I=3 \cdot 10^{5} \mathrm{Nm}^{2}, r=0.075 \mathrm{~m}, L=10, \quad s=2 \mathrm{~m}$, $C_{d}=0, \quad J=\rho I=0.24 \mathrm{~kg} \mathrm{~m}, S=2 \cdot 10^{8} \mathrm{~N}, m_{b}=300 \mathrm{~kg} / \mathrm{m}$ and the observation point $\tilde{x}$ can be chosen arbitrarily. It is assumed $\tilde{x}=0$ for numerical examples. 


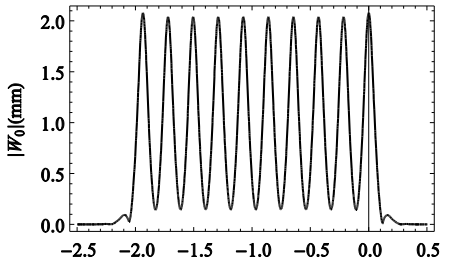

(a)

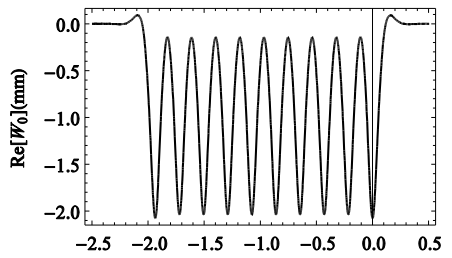

(d)

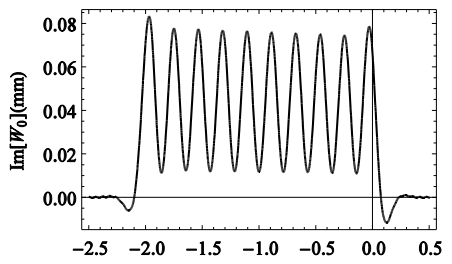

(g)

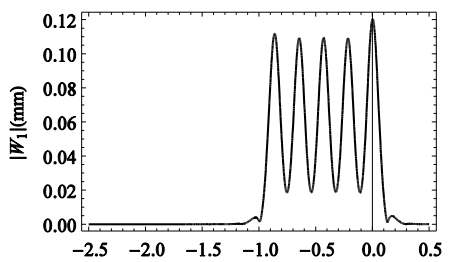

(b)

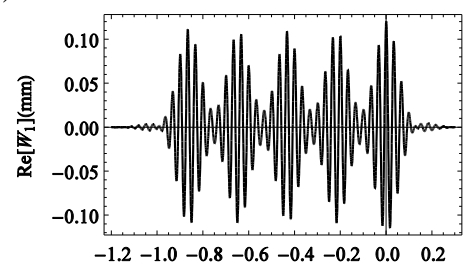

(e)

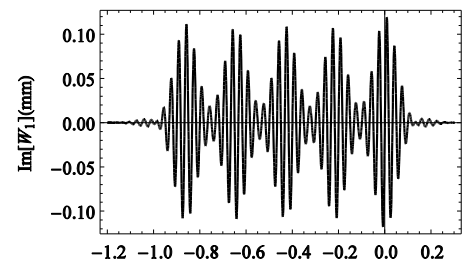

(h)

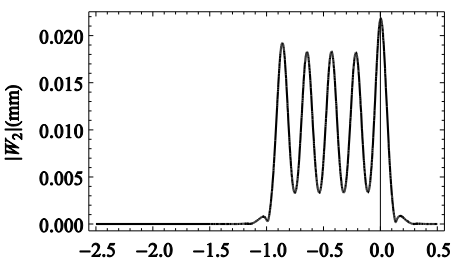

(c)

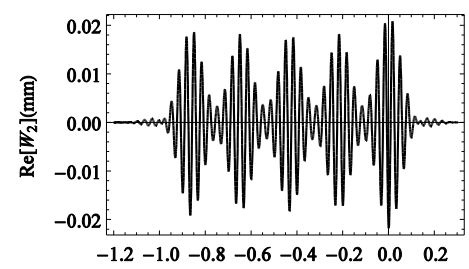

(f)

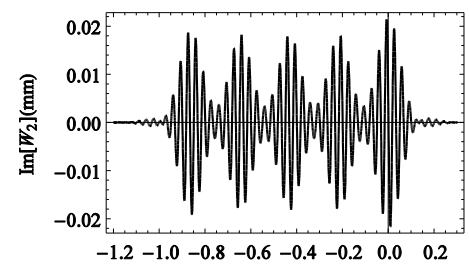

(i)

$\mathrm{t}(\mathrm{s})$

Fig. 3. Terms of Adomian's decomposition for the vertical displacement: $f_{\Omega}=10 \mathrm{~Hz}, V=10 \mathrm{~m} / \mathrm{s}$.

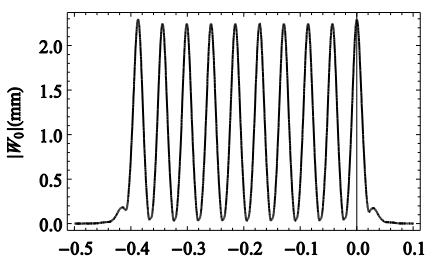

(a)

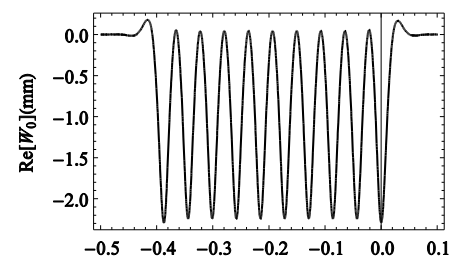

(d)

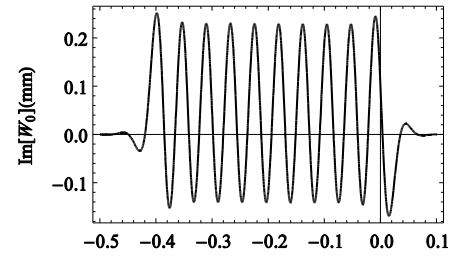

(g)

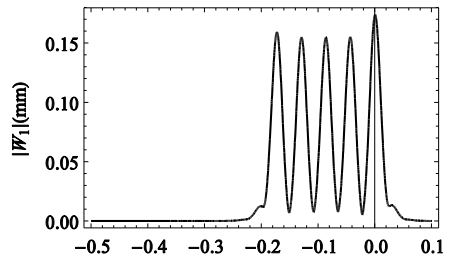

(b)

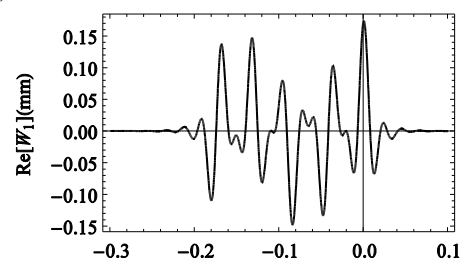

(e)

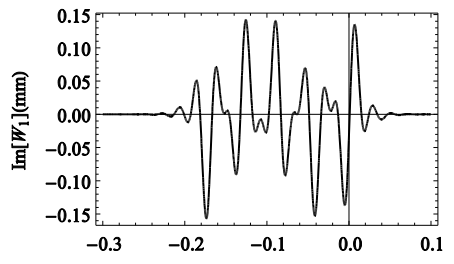

(h)

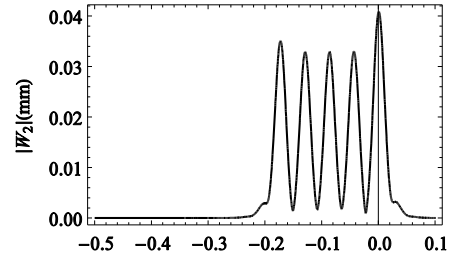

(c)

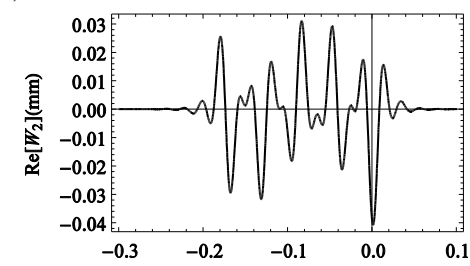

(f)

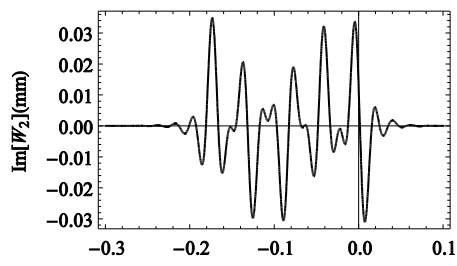

(i) $\mathrm{t}(\mathrm{s})$

Fig. 4. Terms of Adomian's decomposition for the vertical displacement: $f_{\Omega}=10 \mathrm{~Hz}, V=50 \mathrm{~m} / \mathrm{s}$. 


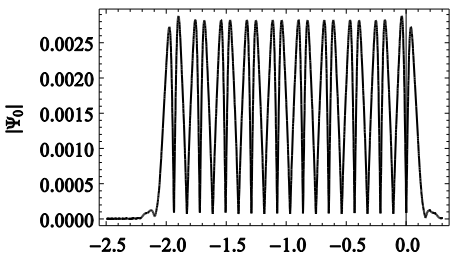

(a)

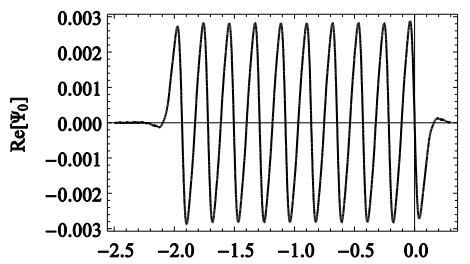

(d)

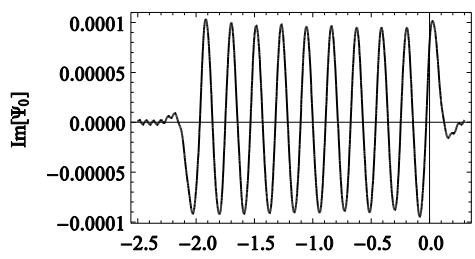

(g)

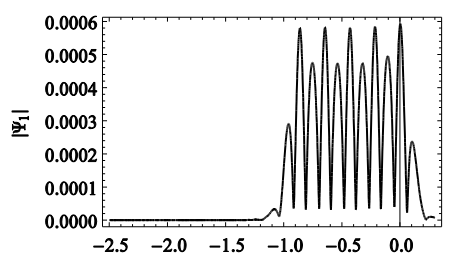

(b)

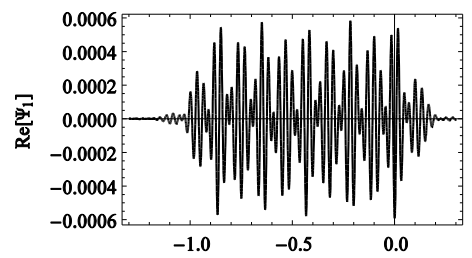

(e)

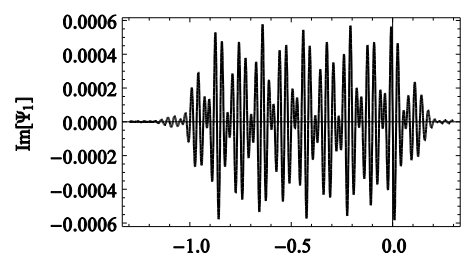

(h)

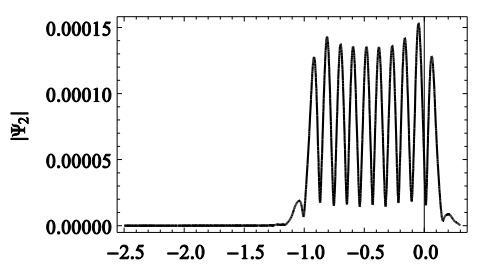

(c)

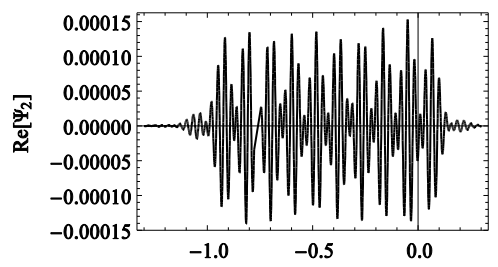

(f)

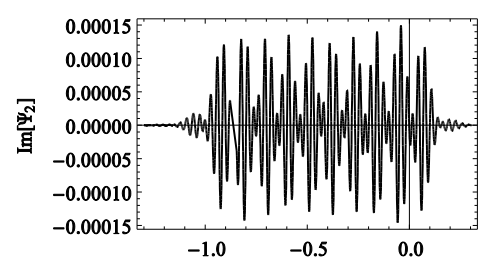

(i)

$\mathbf{t}(\mathbf{s})$

Fig. 5. Terms of Adomian's decomposition for the angular rotation: $f_{\Omega}=10 \mathrm{~Hz}, V=10 \mathrm{~m} / \mathrm{s}$.

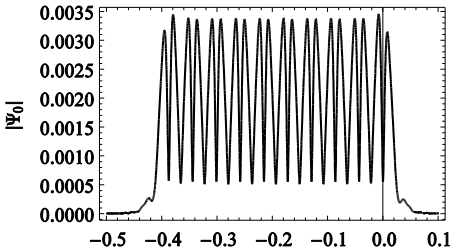

(a)

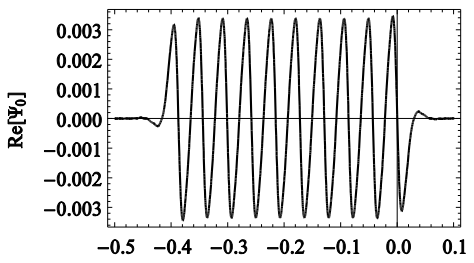

(d)

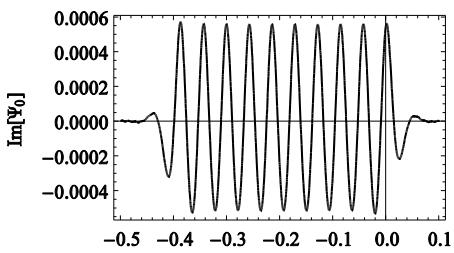

(g)

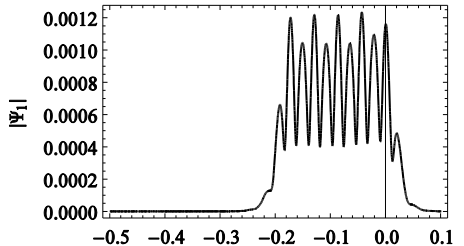

(b)

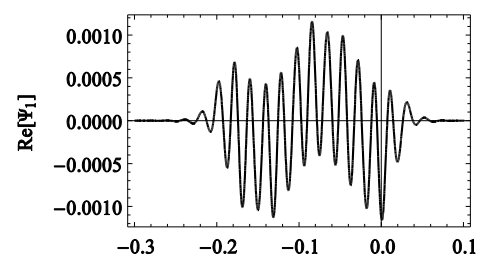

(e)

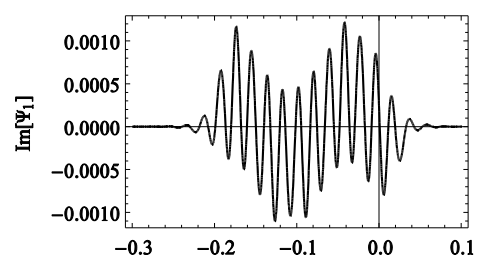

(h)

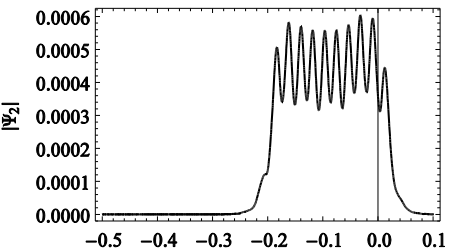

(c)

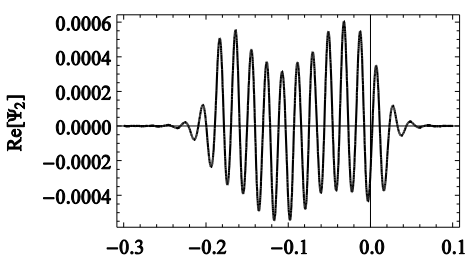

(f)

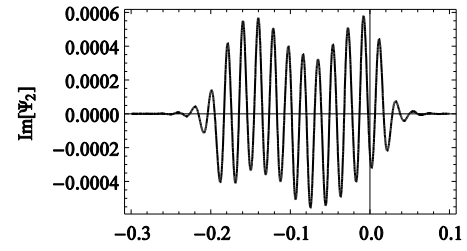

(i) $\mathrm{t}(\mathrm{s})$

Fig. 6. Terms of Adomian's decomposition for the angular rotation: $f_{\Omega}=10 \mathrm{~Hz}, V=50 \mathrm{~m} / \mathrm{s}$. 
Figures 3-6 show the characteristics of a few first terms of Adomian series (12) (i.e. $W_{j}(x, t)=w_{j}(\tilde{x}-V t) e^{i \Omega t}$ and $\Psi_{j}(x, t)=\psi_{j}(\tilde{x}-V t) e^{i \Omega t}$ for $\left.j=0,1,2\right)$. One can see decreasing influence of those terms on the level of vibrations with increasing order $n$ of Adomian's approximation $S_{n}{ }^{w}(x)=S_{n}{ }^{w}(\tilde{x}-V t)$ and $S_{n}{ }^{\psi}(x)=S_{n}{ }^{\psi}(\tilde{x}-V t)$. Therefore the higher order terms of Adomian series $(n>2)$ can be usually neglected. The effect of nonlinearity on the vibratory characteristics of the system becomes stronger for higher velocities with possible the strongest impact in the area of critical values. These features can be observed for both the vertical displacement (Figs 3 and 4) and the angular rotation (Figs 5 and 6). The angular rotation $\Psi$ is more sensitive for a nonlinear factor influence and might need a higher order of approximation for a precise solution.

It is of interest to examine the extreme values of the system response [20-22]. One should note that the real and imaginary parts are not as readable for nonlinear models as for linear case. All representations of the nonlinear solution can be analyzed, i.e. its real part $\operatorname{Re}(w)$, the imaginary part $\operatorname{Im}(w)$ and the modulus $|w|=\sqrt{\operatorname{Re}(w)^{2}+\operatorname{Im}(w)^{2}}$. Even so, its modulus gives information about how strong is the response of the system in time history. Therefore the system investigation can be carried out by analysing so called "the maximal response" represented by the modulus of the complex solution [20,22]. Nevertheless, the real and the imaginary parts of the response can be used for the analysis of mathematical features of the solution.

For some systems of parameters, a nonlinear part of Adomian's solution has significant importance for the response analysis. Figure 7 shows that the beam response for a nonlinear foundation becomes stronger even for relatively low frequencies, compared with a linear case.

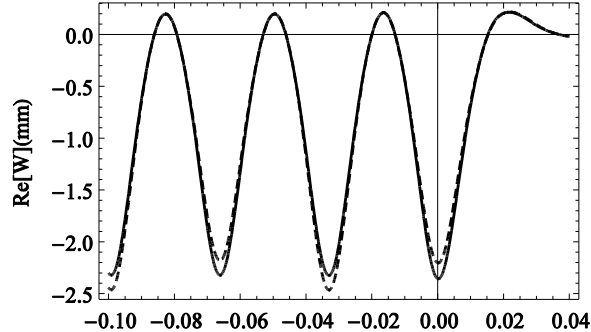

(a)

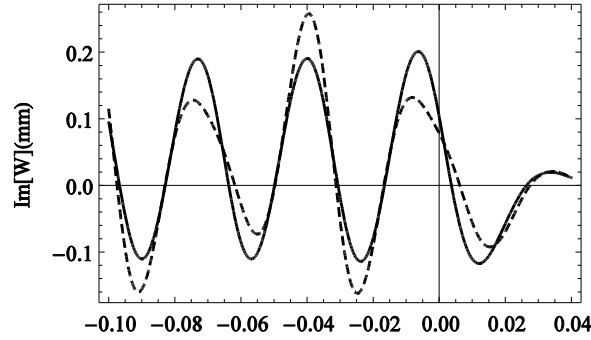

(c)

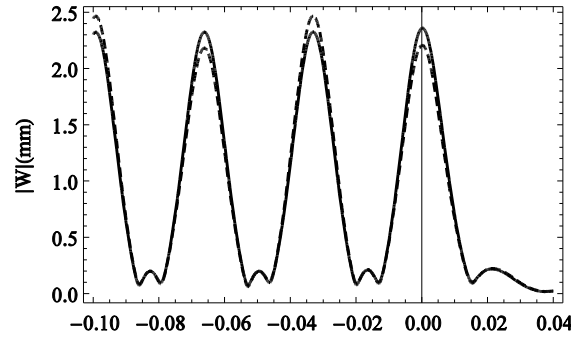

(e)

$\mathrm{t}(\mathrm{s})$ (b)

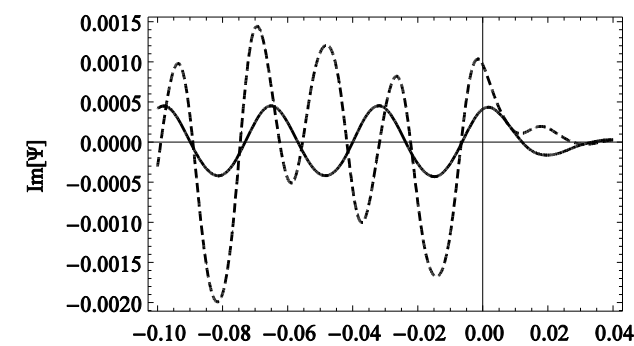

(d)

(f)
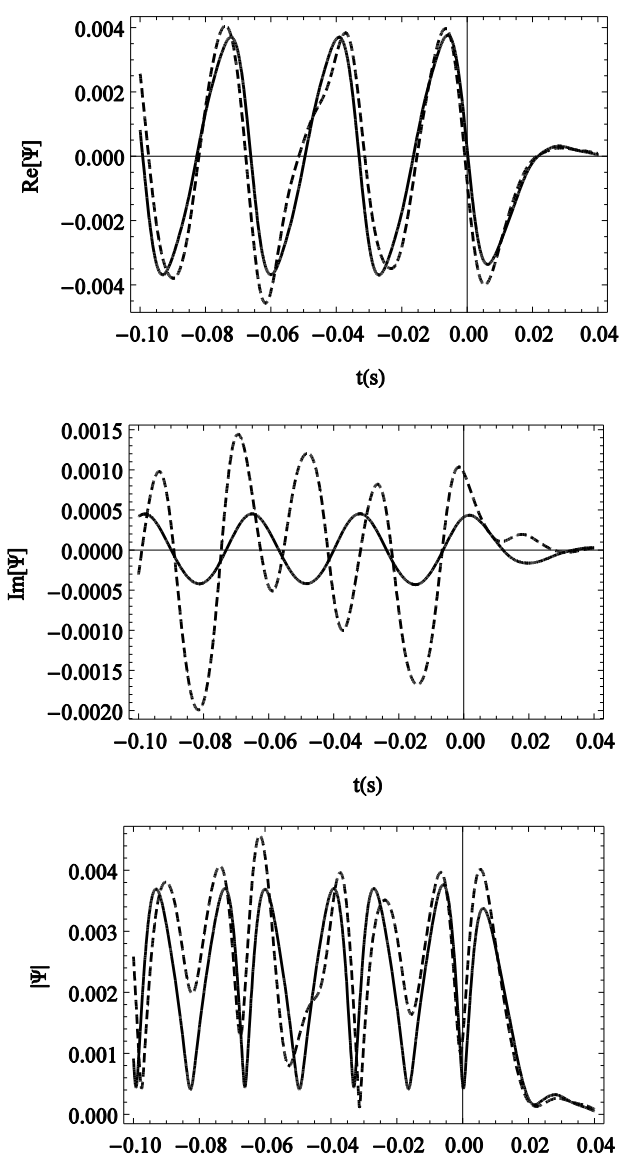

$\mathrm{t}(\mathrm{s})$

$\mathrm{t}(\mathrm{s})$

$\mathrm{t}(\mathrm{s})$

Fig. 7. A comparison of linear and nonlinear response ( $f_{\Omega}=5 \mathrm{~Hz}, V=65 \mathrm{~m} / \mathrm{s}$ ): (a), (c), (e) $W_{0}-$ solid line, $S_{5}{ }^{W}-$ dashed line; (b), (d), (f) $\Psi_{0}$ - solid line, $S_{5}^{\Psi}-$ dashed line. 


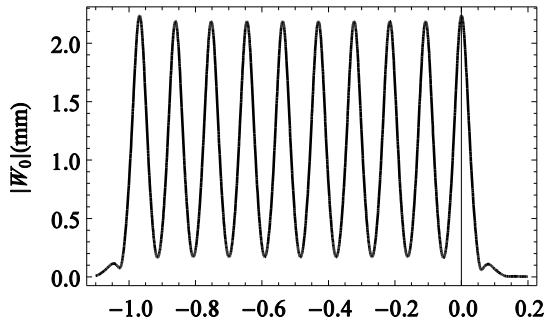

(a)

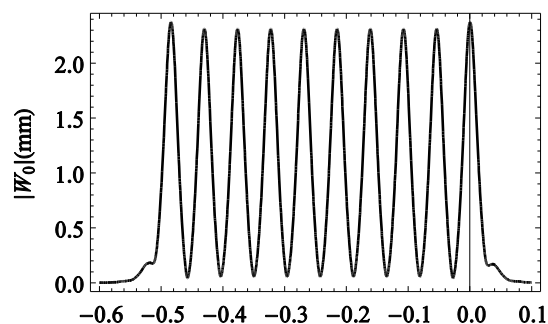

(c)

$\mathrm{t}(\mathrm{s})$

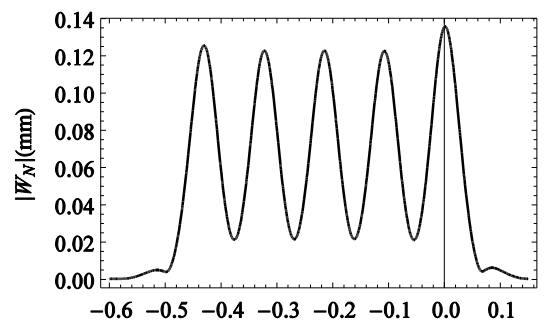

(b)

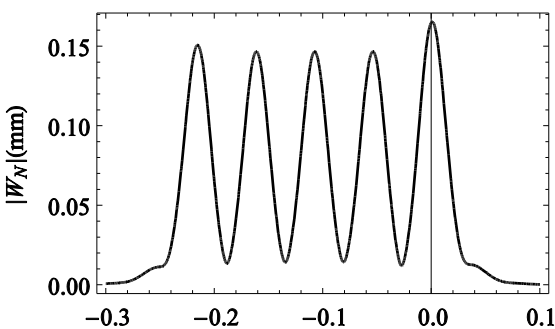

(d)

Fig. 8. The maximum displacement ( $f_{\Omega}=15 \mathrm{~Hz}$,): (a)-(b) $V=20 \mathrm{~m} / \mathrm{s}$; (c)-(d) $V=40 \mathrm{~m} / \mathrm{s}$.

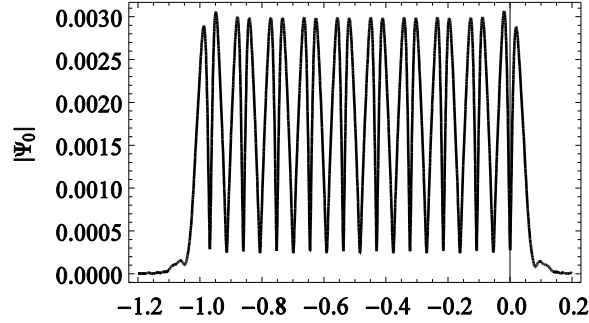

$\mathrm{t}(\mathrm{s})$

(a)

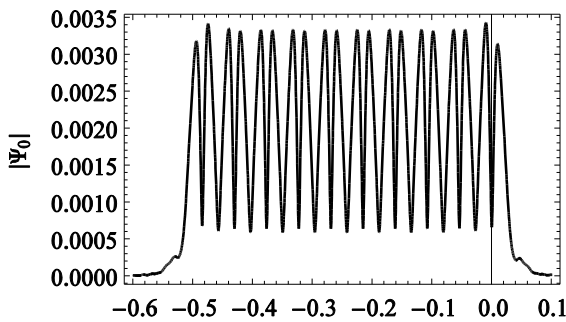

(c) $\mathrm{t}(\mathrm{s})$

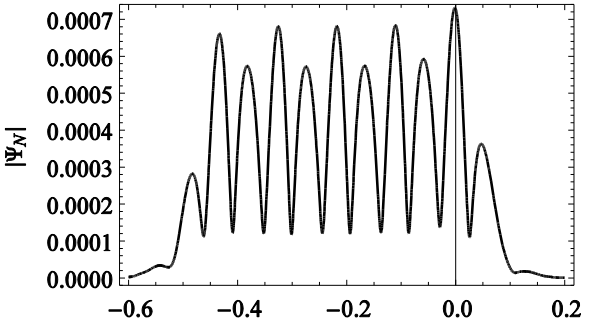

(b)

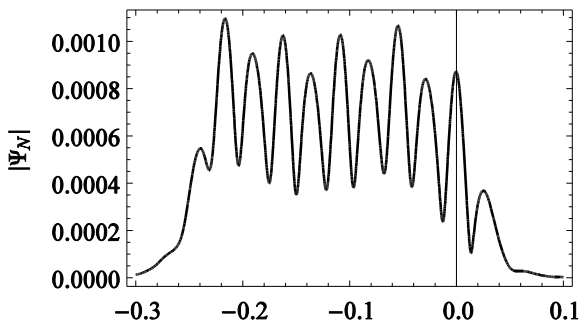

(d) $\mathrm{t}(\mathrm{s})$

$\mathrm{t}(\mathrm{s})$

Fig. 9. The maximum displacement ( $f_{\Omega}=15 \mathrm{~Hz}$,): (a)-(b) $V=20 \mathrm{~m} / \mathrm{s}$; (c)-(d) $V=40 \mathrm{~m} / \mathrm{s}$.

Figures 8 and 9 compare linear terms $W_{0}, \Psi_{0}$ of solution with their approximate nonlinear parts $W_{N}(x, t)=$ $\left(S_{5}{ }^{w}(x)-w_{0}(x)\right) e^{i \Omega t}$ and $\Psi_{N}(x, t)=\left(S_{5}{ }^{\psi}(x)-\psi_{0}(x)\right) e^{i \Omega t}$, showing even $25 \%$ of participation of a nonlinear component in the value of the maximal response (Fig. 9).

The vibrations of the beam are observed in a relatively long time after the load reaches the observation point $\tilde{x}=0$. This feature, coming from the type of applied load, is different from those observed for a single distributed load harmonically varying in time [17]. One should note that the time zero is fixed at the moment when the centre of 
last term of $P(\tilde{x}, t)$ is passing by the observation point $\tilde{x}=0$. It means that the time $t_{0}$ when the load reaches the observation point is negative; e.g. $t_{0} \approx-1 \mathrm{~s}$ for the velocity $V=20 \mathrm{~m} / \mathrm{s}$ (Fig. 8a, see also Fig. 2).

One can observe that the nonlinear terms $W_{N}$ and $\Psi_{N}$ become significantly different from zero after a relatively long time from the point $t_{0}$ and vanish quickly when the load passes the observation point (Figs $3 \mathrm{~b}, 3 \mathrm{c}-6 \mathrm{~b}, 6 \mathrm{c}$ and $8 \mathrm{~b}, 8 \mathrm{~d}, 9 \mathrm{~b}, 9 \mathrm{~d})$. The physical interpretation of this phenomenon, observed for the considered system of parameters, is left as an open problem.

The performed simulations show that the nonlinear factor influences stronger the angular rotation than the vertical vibration (e.g. Figs 7-9). The areas of stability for the obtained solution must be found in order to carry out further analysis and the methodology applied in this paper allows to estimate them numerically, considering the convergence conditions (14)-(15).

One should note that the applied method allows to obtain an approximate solution for the considered nonlinearequation (Eq. (1)). In order to apply effectively the developed methodology, a number of approximating assumptions are made. The exponential term representing harmonic variations in time is added to the distributed load (Eq. (3)) [4] and a linear hysteretic damping instead of the viscous one is considered for a viscoelastic foundation (Eq. (1)) $[21,22]$. Also the Coiflet-based approximation method involves complex numbers and it is applied to the nonlinear term (Eq. (1)). Therefore the real part of the solution might not match exactly the physical response. The physical interpretation of the obtained solution should be considered very carefully. This kind of analysis is an open problem and it is left for further investigations.

\section{Conclusions}

The paper presents a new solution for a dynamic response of the Timoshenko beam resting on a nonlinear viscoelatic foundation and subjected to a moving load represented by a series of distributed loads harmonically varying in time. The developed method of solution using the Adomian's decomposition combined with the wavelet-based approximation of Coiflet type leads to the solution allowing an effective parametric analysis of the system. The use of Timoshenko beam model allowed to analyse the angular rotation of the beam that appeared more sensitive to the considered nonlinearity than the vertical vibrations. The performed simulations show strong efficiency of the wavelet-based approach compared with numerical methods. The presented method can be applied for a detailed analysis of similar nonlinear models and the obtained results can be used as a benchmark for other solutions.

A strong influence of a nonlinear factor on the beam response for some sets of parameters confirms a necessity of investigations of nonlinear dynamic models.

\section{References}

[1] L. Fryba, Vibrations of Solids and Structures under Moving Loads, Thomas Telford Ltd., London, 1999.

[2] T. Dahlberg, Dynamic interaction between train and nonlinear railway model, in: Proceedings of Fifth International Conference on Structural Dynamics, Munich, 2002.

[3] T.X. Wu and D.J. Thompson, The effects of track non-linearity on wheel/rail impact, Proc Inst Mech Eng Part F: Rail Rapid Transit, 218:1-12, 2004.

[4] M.H. Kargarnovin, D. Younesian, D.J. Thompson and C.J. Jones, Response of beams on nonlinear viscoelastic foundations to harmonic moving loads, Computers and Structures 83 (2005), 1865-1877.

[5] P. Koziol, Wavelet analysis of multilayered ground vibrations as a result of high speed trains, CC2009, Proceedings of the Twelfth International Conference on Civil, Structural and Environmental Engineering Computing, Funchal, Madeira, 1-4 September, 2009.

[6] M.F.M. Hussein and H.E.M. Hunt, Modelling of floating-slab tracks with continuous slabs under oscillating moving loads, Journal of Sound and Vibration 297 (2006), 37-54.

[7] G. Adomian, Nonlinear Stochastic Systems Theory and Application to Physics, Kluwer Academic Publishers, Dordrecht, 1989.

[8] P. Koziol, Wavelet approach for the vibratory analysis of beam-soil structures: Vibrations of dynamically loaded systems, VDM Verlag Dr. Müller, Saarbrucken, 2010.

[9] A. Pourdarvish, A reliable symbolic implementation of algorithm for calculating Adomian polynomials, Applied Mathematics and Computation 172 (2006), 545-550.

[10] Z. Hryniewicz and P. Koziol, Wavelet approach for analysis of dynamic response of Timoshenko beam on random foundation, ICSV16, The Sixteenth International Congress on Sound and Vibration, Kraków, 5-9 July, 2009. 
[11] M.M. Hosseini and H. Nasabzadeh, On the convergence of Adomian decomposition method, Applied Mathematics and Computation 182(1) (2006), 536-543.

[12] P. Koziol and C. Mares, Wavelet approach for vibration analysis of fast moving load on a viscoelastic medium, Shock and Vibration 17(4,5) (2010), 461-472.

[13] S. Mallat, A Wavelet Tour of Signal Processing, Academic Press, 1998.

[14] J. Wang, Y. Zhou and H. Gao, Computation of the Laplace inverse transform by application of the wavelet theory, Communications in Numerical Methods in Engineering 19 (2003), 959-975.

[15] Daubechies, Orthonormal bases of compactly supported wavelets II. Variations on a theme, SIAM J Math Anal 24 (1993), $499-519$.

[16] L. Monzon, G. Beylkin and W. Hereman, Compactly supported wavelets based on almost interpolating and nearly linear phase filters (coiflets), Applied and Computational Harmonic Analysis 7 (1999), 184-210.

[17] P. Koziol and Z. Hryniewicz, Wavelet based solution for vibrations of a beam on a nonlinear viscoelastic foundation due to a moving load, XII Symposium "The Influence of Vibrations on Environment”, Kraków - Janowice, 2010.

[18] S.-M. Kim, Stability and dynamic response of Rayleigh beam-columns on an elastic foundation under moving loads of constant amplitude and harmonic variation, Engineering Structures 27 (2005), 869-880.

[19] I. Coskun, Non-linear vibrations of a beam resting on a tensionless Winkler foundation, Journal of Sound and Vibration 236(3) (2000), 401-411.

[20] L. Sun, A closed-form solution of beam on viscoelastic subgrade subjected to moving loads, Computers and Structures 80 (2002), 1-8.

[21] S.-M. Kim, Vibration and stability of axial loaded beams on elastic foundation under moving harmonic loads, Engineering Structures $\mathbf{2 6}$ (2004), 95-105.

[22] S.-M. Kim and Y.-H. Cho, Vibration of dynamic buckling of shear beam-columns on elastic foundation under moving harmonic loads, International Journal of Solids and Structures 43 (2006), 393-412. 

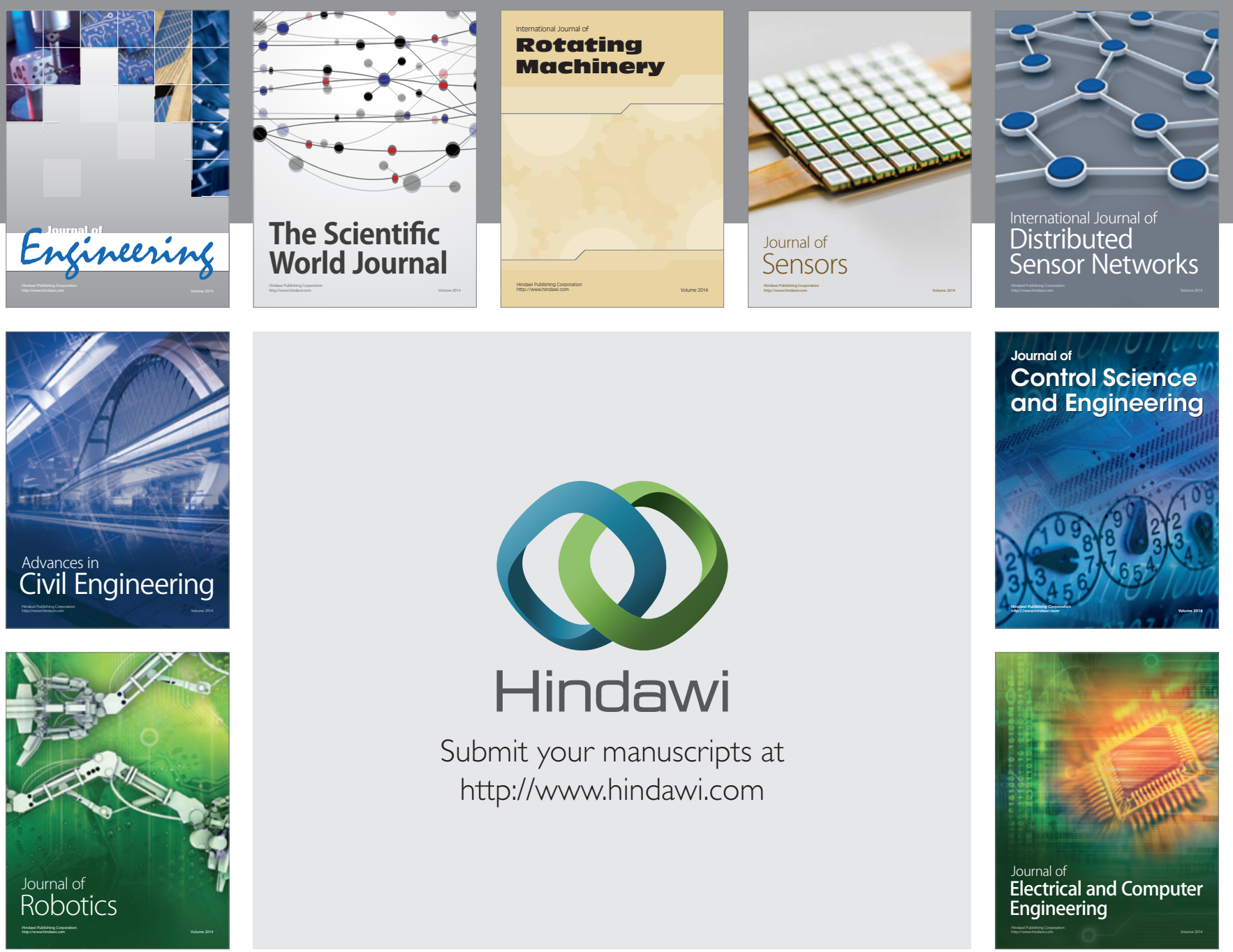

Submit your manuscripts at

http://www.hindawi.com
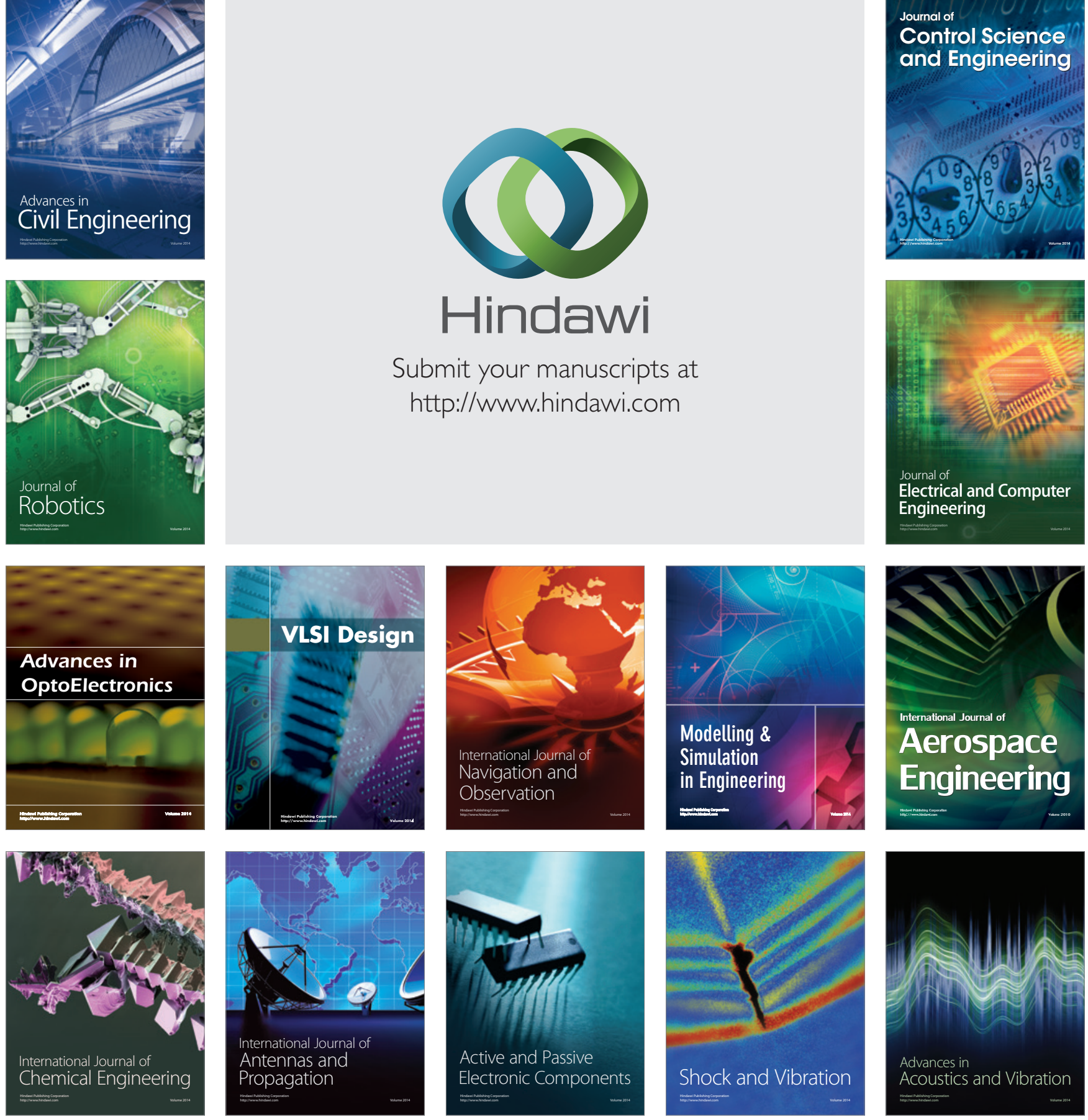